\title{
La renta mínima como instrumento para combatir la pobreza. El caso de la Comunidad Valenciana
}

\author{
Amadeo Fuenmayor Fernández \\ Rafael Granell Pérez \\ Teresa Savall Morera \\ Universitat de València. Departament d'Economia Aplicada \\ amadeo.fuenmayor@uv.es; rafael.granell@uv.es; teresa.savall@uv.es
}

Recepción: 04-07-2019

Aceptación: 16-04-2020

Publicación: 06-05-2020

\section{Resumen}

Recientemente, la Comunidad Valenciana ha reformado la renta mínima y ha instaurado la denominada Renta Valenciana de Inclusión (RVI). En este trabajo realizamos una evaluación de este programa utilizando técnicas de microsimulación a partir de microdatos proporcionados por la Administración valenciana.

Se hace una descripción del nuevo programa respecto a la anterior Renta Garantizada de Ciudadanía (RGC), que ha mostrado problemas de agotamiento, y se señalan las diferencias más relevantes.

A partir de los datos existentes, calculamos el coste presupuestario estimado de la RVI y los efectos de este programa en términos de pobreza y desigualdad. Aunque el gasto se incrementará respecto a la antigua RGC, los cambios en el diseño implicarán mejoras muy importantes en términos de pobreza y desigualdad.

Palabras clave: renta mínima de inserción; microsimulación; pobreza; desigualdad

Abstract. Minimum income as an instrument to fight against poverty. The case of Valencian region

Recently, the Valencian Community has reformed the minimum income, establishing the so-called Valencian Inclusion Income (VII). In this work we performed an evaluation of this program, using microsimulation techniques from microdata provided by the Valencian administration.

A description of the new program is made regarding the previous Guaranteed Citizenship Income (GCI) that has shown problems of exhaustion, pointing out the most relevant differences.

From the existing data, we calculate the estimated budgetary cost of the VII and the effects of this program in terms of poverty and inequality. Although spending will increase with respect to the old GCI, the changes in the design will imply very important improvements in terms of poverty and inequality.

Keywords: minimum insertion income; microsimulation; poverty; inequality 


\section{Sumario}

1. Introducción 4. Estimación de los efectos sobre

2. El anterior programa de rentas mínimas la pobreza y la desigualdad de la Comunidad Valenciana: la Renta de Garantía de la Ciudadanía (RGC)

3. El nuevo programa de rentas mínimas de la CV: la Renta Valenciana 5. Conclusiones

Financiación Referencias bibliográficas de Inclusión (RVI)

\section{Introducción}

Los niveles de pobreza y exclusión social se han incrementado de forma alarmante en España durante el periodo de recesión: el indicador $\mathrm{AROPE}^{1}$ ha pasado del $23,8 \%$ al 29,2 \% entre 2008 y 2014 , y ha aumentado también la diferencia entre España y la media de los países europeos. En la Comunidad Valenciana (CV), la situación social es más crítica, pues dicho indicador ha pasado de un $27,5 \%$ en 2008 a un $34,7 \%$ en 2014 . Aunque estas cifras han mejorado ligeramente durante los últimos años —se han situado en el 26,1\% en España y en el 30,2 \% en la CV en 2018-, todavía estamos muy alejados de los niveles de pobreza previos a la recesión. No obstante, el componente más alarmante del indicador, la carencia material severa, ha mejorado durante los últimos años en la CV y ha pasado del $11,3 \%$ en 2014 al 2,9 \% en 2018, con una reducción sobre todo durante el último año.

Otro problema, considerado una nueva forma de pobreza, que ha puesto en situación de vulnerabilidad a muchas personas es la existencia de trabajadores y pensionistas pobres. A pesar de contar con contrato de trabajo, 691.564 personas cobraban en la CV un salario inferior al salario mínimo interprofesional (SMI) durante 2015, mientras que el 20,31 \% de los pensionistas percibían una pensión no contributiva inferior a la pensión contributiva mínima.

En estas situaciones se pone de manifiesto la debilidad de los sistemas de protección social vigentes y, por lo tanto, es necesario analizar su diseño y sus características tanto desde el ámbito científico como desde la esfera política, para proponer cambios que se traduzcan en mejoras de la situación socioeconómica de la sociedad (Laparra y Ayala, 2009). Concretamente, los programas de rentas mínimas son los instrumentos diseñados para reducir estos problemas, por lo que serán nuestro objeto de estudio. En este trabajo queremos profundizar en el análisis de los programas de rentas mínimas de inserción como instrumento para combatir la pobreza.

Concretamente, se presenta el estudio del caso de la Comunidad Valenciana, con el que pretendemos aportar evidencias empíricas a partir del análisis de

1. Indicador que cuantifica la proporción de personas en riesgo de pobreza o exclusión social. Ver: <http://ec.europa.eu/eurostat/statistics-explained/index.php/Glossary:At_risk_of_poverty_or_social_exclusion_(AROPE)>. 
datos referentes al programa de rentas mínimas vigente hasta 2017, la Renta Garantizada de Ciudadanía (RGC), y también de la evaluación del nuevo programa de rentas mínimas aprobado por la Generalitat Valenciana, la Renta Valenciana de Inclusión (RVI).

La hipótesis desde la que se parte, basada en estudios anteriores, es que la mayoría de los programas autonómicos de rentas mínimas en España no cumplen el objetivo de reducir los niveles de pobreza, en gran medida debido a la baja cobertura de estas prestaciones respecto a la población en riesgo y a la baja cuantía de las prestaciones, que en muchos casos no consigue superar el umbral de la pobreza (Lavia et al., 2014; Rodríguez-Cabrero, 2009; Laparra, 2004; Moreno et al., 2003). En el caso de la CV, existen indicios de que el programa vigente hasta 2017 (RGC) presenta problemas de diseño que no permiten mejorar la situación de pobreza. Se espera que los cambios propuestos en el nuevo programa (RVI) mejoren la efectividad en el objetivo de lucha contra la pobreza.

Respecto a la estructura del artículo, en primer lugar se describe el programa de rentas mínimas de la Comunidad Valenciana vigente hasta 2017, la RGC, contextualizado en el sistema de garantía de ingresos mínimos en España. A continuación, como primeros resultados, se analizan las limitaciones que presenta este programa y que dificultan su eficacia en términos de mejora de los niveles de pobreza. Posteriormente, se especifica la metodología y el proceso de cálculo utilizados para una evaluación del nuevo programa de rentas mínimas planteado por el Gobierno valenciano, en la que se han estimado la evolución en términos de gasto, beneficiarios y cuantía media de la nueva RVI. Finalmente, se profundiza en el aspecto central del artículo, la medición y la comparación de los niveles de pobreza y desigualdad, con la cuantificación del impacto del nuevo programa de rentas mínimas.

\section{El anterior programa de rentas mínimas de la Comunidad Valenciana: la Renta de Garantía de la Ciudadanía (RGC)}

\subsection{Contextualización: el sistema de garantía de ingresos minimos}

Antes de centrar la atención en los programas de rentas mínimas de inserción (RMI), es interesante contextualizarlos dentro del sistema de garantía de ingresos mínimos que existe en España. Este sistema lo configuran distintos mecanismos que tratan de garantizar unos ingresos mínimos cuando la protección por parte de la Seguridad Social no es suficiente o es inexistente.

Del análisis de Rodríguez-Cabrero (2009) se puede destacar que las principales características del sistema español de garantía de ingresos mínimos son:

- Desde una perspectiva institucional, se distingue entre las prestaciones contributivas, gestionadas por la Seguridad Social, y las prestaciones asistenciales, que a su vez pueden ser gestionadas directamente por el estado - complementos de mínimos y algunas prestaciones por desempleo-, de forma compartida con las comunidades autónomas (CCAA) — pensiones 
no contributivas - o a través de las propias CCAA — rentas mínimas de inserción.

- Desde la perspectiva de la naturaleza de la protección social, los diferentes programas se estructuran en función de los posicionamientos ante el mercado de trabajo, en que la participación, presente o pasada, en el mercado de trabajo es el criterio a partir del cual se organiza el acceso a la protección de rentas. Por una parte, estarán los complementos de mínimos como pensiones contributivas y, por el otro, las prestaciones no contributivas, que se engloban en tres modalidades: las prestaciones sociales relacionadas con el desempleo, pensiones no contributivas (por jubilación y por invalidez) y los programas de rentas mínimas de inserción de las CCAA.

Otra clasificación de estos programas la propone Laparra (2004) en función de si el perceptor se encuentra potencialmente activo en el mercado de trabajo y según el grado de protección recibido por parte de la Seguridad Social.

En la literatura (Rodríguez-Cabrero, 2009; Ayala et al., 2016), se identifican los principales problemas que presenta el sistema de garantías de ingresos mínimos, como son la baja intensidad protectora, la existencia de lagunas protectoras (parte de las personas sin hogar, personas sin residencia legal, personas que acceden por primera vez al mercado de trabajo), la incompatibilidad entre programas y la imposibilidad de alcanzar niveles de renta por encima del umbral de pobreza relativa por parte de los perceptores. Son estos estudios previos los que motivan y dan soporte al análisis que se propone a continuación.

En general, los programas de garantía de mínimos van dirigidos a colectivos con un nivel muy bajo de recursos y, concretamente, cada uno de ellos exige que se cumplan distintos requisitos para beneficiar a colectivos concretos.

Centrándonos en nuestro objeto de estudio, los programas de RMI contemplan una prestación económica para aquellas personas que tienen unos ingresos inferiores a una cantidad mínima prefijada y que, por tanto, están en una situación de pobreza (Arriba, 2014; Urteaga, 2011). Para el cálculo de la prestación, se comprueba si las rentas del hogar son inferiores a la cantidad teórica establecida. En caso afirmativo, la cuantía de la prestación será el resultado de restar a la cantidad teórica las rentas recibidas previamente por el hogar.

Tabla 1. Programas de garantía de mínimos en el sistema español de protección social

\begin{tabular}{lll}
\hline & $\begin{array}{l}\text { Población potencialmente } \\
\text { activa (edad de trabajar, } \\
\text { no discapacitados) }\end{array}$ & $\begin{array}{l}\text { Población potencialmente no } \\
\text { activa (mayores y discapacitados) }\end{array}$ \\
\hline $\begin{array}{l}\text { Protección insuficiente } \\
\text { de la Seguridad Social } \\
\text { contributiva }\end{array}$ & $\begin{array}{l}\text { Subsidios asistenciales por } \\
\text { desempleo («complementarias», } \\
\text { subsidio agrario y renta activa) }\end{array}$ & $\begin{array}{l}\text { Complementos de mínimos } \\
\text { de pensiones }\end{array}$ \\
\hline $\begin{array}{l}\text { No protegidos por } \\
\text { la Seguridad Social } \\
\text { contributiva }\end{array}$ & $\begin{array}{l}\text { Rentas mínimas de inserción } \\
\text { «salarios sociales» con diversos } \\
\text { nombres) }\end{array}$ & $\begin{array}{l}\text { Pensiones no contributivas } \\
\text { (y pensiones asistenciales y } \\
\text { subsidios LISMI a extinguir) }\end{array}$ \\
\hline
\end{tabular}

Fuente: Laparra (2004). 
En el contexto español, los programas de RMI se empiezan a desarrollar a finales de los años 80 , fruto de reivindicaciones de sindicatos y organizaciones sociales y religiosas. Al inicio, se abogaba por un sistema centralizado, común para toda España, pero ante la falta de desarrollo, los gobiernos regionales trataron de rellenar este vacío de manera un tanto improvisada y sin ningún mecanismo de coordinación, lo que provocó diferencias muy importantes en requisitos, cuantías y derechos (Arriba, 1999).

La primera comunidad autónoma en poner en marcha un programa de RMI fue el País Vasco (año 1989), cuya cobertura, efectividad y cuantía destacan sobre el resto, hasta el punto de poder equipararse a los programas existentes en otros países europeos (Lavia et al., 2014). Sin embargo, los programas de la mayoría de comunidades autónomas no cumplen los requisitos de un programa de RMI tal y como se define a nivel europeo (Ayala, 2004b; Bergantiños et al., 2017), dado que no consiguen ofrecer una cobertura adecuada para superar el nivel de pobreza.

A día de hoy, existen 19 sistemas distintos de RMI, tantos como comunidades autónomas y ciudades autónomas, que son regulados y financiados por cada una de ellas. Todas estas rentas autonómicas tienen características en común, pero también múltiples diferencias.

Las semejanzas se refieren fundamentalmente a los objetivos que persiguen estos sistemas: ayudar financieramente a personas o familias que se encuentran en una situación muy desfavorable actuando como una renta mínima garantizada; e insertar laboral y socialmente a los perceptores de estas rentas para que puedan salir de la situación de pobreza y exclusión en la que se encuentran. Mientras que las diferencias entre los distintos sistemas de RMI se ponen de manifiesto en los requisitos de acceso a los beneficiarios (CES, 2017) y en las cuantías concedidas a los mismos (Fuenmayor et al., 2011).

Respecto a la evolución reciente de los programas de RMI en España, cabe destacar los resultados de estudio de Bergantiños et al. (2017), en el que se pone de manifiesto que la respuesta ante la crisis de las distintas CCAA no ha sido homogénea en la gestión de las RMI, aunque en general se ha producido una cierta mejoría de los distintos programas de RMI, independientemente de su situación de partida. También se destaca que persiste la falta de equidad territorial y las tasas de baja cobertura de este tipo de mecanismos, ya que la gran mayoría de CCAA cubren menos del $10 \%$ de la población en riesgo.

\subsection{La renta garantizada de ciudadanía}

El sistema de RMI vigente hasta la entrada en vigor del nuevo programa en la Comunitat Valenciana se denominaba renta de garantía de la ciudadanía (RGC) y se aprobó en el año 2007 a través de la Ley 9/2007 de la Generalitat Valenciana. $^{2}$

2. Ley 9/2007, de 12 de marzo, de la Generalitat, de Renta Garantizada de Ciudadanía de la Comunidad Valenciana (DOGV núm. 5475 de 22.03.2007). 
Entre sus características principales, cabe destacar que es un sistema de prestaciones al que pueden acceder personas con residencia legal durante dos años en la $\mathrm{CV}$, que tengan entre 25 y 65 años y posean unos ingresos mensuales inferiores a $385 €$ (en el caso de unidades familiares unipersonales), con un periodo máximo

Tabla 2. Perfil de los titulares perceptores de la RGC en la CV, 2015

\begin{tabular}{|c|c|c|}
\hline & & Porcentaje \\
\hline \multirow[t]{2}{*}{ Ingresos previos } & Sin ingresos & $68,2 \%$ \\
\hline & Con ingresos & $31,8 \%$ \\
\hline \multirow[t]{2}{*}{ Género } & Hombre & $31,2 \%$ \\
\hline & Mujer & $68,8 \%$ \\
\hline \multirow[t]{5}{*}{ Tramos de edad } & Menor de 25 & $4,8 \%$ \\
\hline & Entre 25 y 34 & $23,2 \%$ \\
\hline & Entre 35 y 44 & $37,9 \%$ \\
\hline & Entre 45 y 54 & $22,5 \%$ \\
\hline & Mayor de 55 & $11,7 \%$ \\
\hline \multirow[t]{5}{*}{ Estudios } & Otros & $17,5 \%$ \\
\hline & Primaria o inferior & $77,0 \%$ \\
\hline & Secundaria & $0,3 \%$ \\
\hline & Bachiller/ FP medio & $3,8 \%$ \\
\hline & Grado universitario/ FP superior & $1,4 \%$ \\
\hline \multirow[t]{4}{*}{ Tipo de hogar } & Unipersonal & $33,4 \%$ \\
\hline & Monoparental & $39,6 \%$ \\
\hline & Pareja con hijos & $20,7 \%$ \\
\hline & Otros & $6,3 \%$ \\
\hline \multirow[t]{7}{*}{ Tamaño hogar } & 1 & $33,4 \%$ \\
\hline & 2 & $20,6 \%$ \\
\hline & 3 & $20,5 \%$ \\
\hline & 4 & $14,1 \%$ \\
\hline & 5 & $7,5 \%$ \\
\hline & 6 & $2,6 \%$ \\
\hline & $>7$ & $1,3 \%$ \\
\hline \multirow[t]{2}{*}{ Nacionalidad } & Española & $78,7 \%$ \\
\hline & Extranjera & $21,3 \%$ \\
\hline \multirow[t]{5}{*}{ Tipo de actividad } & Trabajo por cuenta propia & $0,2 \%$ \\
\hline & Trabajo por cuenta ajena & $0,7 \%$ \\
\hline & Desempleo & $31,3 \%$ \\
\hline & Jubilación & $0,0 \%$ \\
\hline & Otra clase de inactividad & $67,8 \%$ \\
\hline \multirow[t]{4}{*}{ Inserción laboral } & No se ha participado & $0,0 \%$ \\
\hline & Participación medidas generales & $5,4 \%$ \\
\hline & Participación estrategias intensivas de empleo & $3,3 \%$ \\
\hline & Participación en los dos tipos de actividades & $91,2 \%$ \\
\hline
\end{tabular}

Fuente: elaboración propia a partir de datos proporcionados por la Conselleria de Igualdad y Políticas Inclusivas. 
de percepción de la prestación de seis meses. La cuantía de la prestación tiene un límite máximo de $385 €$ mensuales, que va aumentando según el tamaño familiar. Sin embargo, la prestación realmente percibida se calcula restando a este importe máximo los ingresos previos obtenidos por el solicitante.

El gasto ejecutado destinado a la RGC ha tenido una evolución creciente durante el tiempo y ha pasado de 2,4 millones de euros en 2008 a casi 45 millones de euros en 2016. Este aumento del gasto responde básicamente a un claro incremento del número de perceptores, de 3.561 personas en 2008 a 20.007 en 2016. El gasto anual por beneficiario en la CV se ha mantenido relativamente estable, siempre por debajo de la media española (2015: $760 €$ en la CV frente a $1.492 €$ de media española).

En la tabla 2 se presentan los datos referentes al perfil sociodemográfico de los perceptores de la RGC en el año 2015. De estos resultados cabe destacar que más del $68 \%$ no tienen otro tipo de ingresos, lo que significa que perciben la cuantía máxima de la RGC correspondiente a su tipo de familia; cerca del $70 \%$ de los titulares son mujeres; en cuanto a la distribución por edad, el $38 \%$ corresponde a personas entre 35 y 44 años; un $77 \%$ son personas con estudios primarios o inferiores; los hogares monoparentales representan casi un $40 \%$ del total; una amplia mayoría de los perceptores $(78,7 \%)$ tiene nacionalidad española, aunque cabe destacar que el porcentaje de titulares extranjeros $(21,3 \%)$ es mayor al porcentaje de extranjeros sobre la población total de la CV $(13,1 \%)$; son los inactivos $(67,8 \%)$ y los desempleados $(31,3 \%)$ los que reciben casi la totalidad de la prestación en términos de tipo de actividad; y más del $90 \%$ de los titulares participan en los dos tipos de actividades de inserción contempladas.

\subsection{Limitaciones de la $R G C$}

La literatura destaca una serie de limitaciones comunes a los distintos programas de RMI de las comunidades autónomas (Lavia et al., 2014; RodríguezCabrero, 2009; Laparra, 2004; Moreno et al., 2003; Bergantiños et al., 2017; Riba et al., 2011):

- No existe un reconocimiento del derecho a la prestación por ley, es decir, no se reconoce como un derecho subjetivo del Sistema de Garantía de Rentas. Se consideran prestaciones no garantizadas, supeditadas a la disponibilidad presupuestaria, de manera que el número de concesiones está condicionando al presupuesto anual destinado al programa.

- No se concibe el programa como un doble derecho, por lo que no se garantiza tanto la protección económica como la inserción laboral. Domina una concepción más restrictiva, la contraprestación, según la cual se entiende que los perceptores deben pagar con determinadas obligaciones la deuda que contraen con la sociedad al recibir una prestación económica.

- Bajo nivel de cobertura respecto a los potenciales perceptores, pues dejan fuera de la última red de garantía a personas con graves problemas económicos y de inserción social. 
- Insuficiente cuantía de las prestaciones para alcanzar el nivel de renta del umbral de pobreza, tanto en los casos de individuos como de familias con más miembros, con el problema habitual de infravaloración de las escalas de equivalencia.

- Bajo impacto de las actividades de inserción, producido en parte por la falta de adaptación de los itinerarios a las características individuales de cada caso y también por la baja colaboración con las administraciones locales y con entidades no lucrativas.

- Deficiencias en la forma en la que se gestiona la permanencia en el programa, tanto en términos de tiempo inicial de concesión como de facilidad de renovación.

A continuación, se analizan las limitaciones concretas que presenta la RGC. Estas insuficiencias motivaron la decisión del Gobierno valenciano de revisar el programa.

En primer lugar, la RGC se considera un derecho subjetivo en la propia ley, pero su gestión durante los últimos años evidencia que la concesión de las prestaciones está sujeta a disponibilidad presupuestaria, por lo que las concesiones son limitadas. Además, no existe una concepción doble del derecho, pues en la propia ley se considera que la finalidad es prestar un apoyo económico que permita favorecer la inserción sociolaboral, pero esta no es el objetivo en sí mismo. De hecho, en la ley se considera que participar en los programas de inserción es una obligación, con lo que se reafirma que la inserción se concibe como la contrapartida a la prestación.

Para obtener el nivel de cobertura (Laparra, 2004; Ayala et al., 2016), se contrasta el número de perceptores con el volumen de potenciales beneficiarios. Para obtener el volumen de potenciales beneficiarios, se recurre a la Encuesta de Población Activa (EPA) y se toman aquellos hogares que no disponen de un empleo, ni de una pensión, ni de prestación o subsidio de desempleo. Este colectivo es la población objetivo a la que van dirigidos los programas de rentas mínimas. La cobertura, medida en estos términos, de la RGC en el año 2016 era del 16,68 \%. Este bajo porcentaje confirma que la limitación de bajo nivel de cobertura está presente en el caso de la RGC.

Otra manera de establecer el nivel de cobertura de la RGC es tomar como referencia la población en riesgo de pobreza severa, ${ }^{3}$ pues se considera que el programa de rentas mínimas es la última red de protección social dentro del sistema de garantía de mínimos y por lo tanto cubre las necesidades de la población más desfavorecida. Concretamente, este indicador se construye relacionando el número total de beneficiarios totales del programa (teniendo en cuenta a los miembros del hogar que no son titulares) de la RGC y la población en riesgo de pobreza severa de la Comunidad Valenciana para cada año. Dicha tasa de cobertura de la RGC en los últimos años ha sido creciente, ya que ha pasado del 2,27 \% en 2008

3. Según Eurostat, se considera como umbral de pobreza severa el nivel de renta inferior al $30 \%$ de la renta mediana. 
al $10,90 \%$ en 2015 . Pero pese a la evolución positiva, se considera una tasa baja comparada con los datos de la media española para ese mismo año $(22,62 \%)$.

Otra limitación que presenta la RGC está vinculada a la insuficiencia de la cuantía de las prestaciones para alcanzar el nivel de renta del umbral de pobreza. Ni el importe medio de las prestaciones concedidas en los últimos años ni tampoco la cuantía teórica máxima que se puede conceder con la RGC alcanzan el nivel de renta del umbral de pobreza. Es decir, la RGC no permite a ninguna persona salir de la pobreza, ni siquiera de la pobreza severa. ${ }^{4}$

Por último, hay que destacar el problema de la permanencia en el programa. La RGC, normativamente, contempla un máximo de doce meses de concesión de la prestación, aunque en la práctica este periodo se ha visto reducido a seis meses debido a la limitación presupuestaria. Este es el primer elemento que dificulta el acceso al programa, dado que cada año se tiene que volver a solicitar. Este hecho complica la renovación porque aparecen problemas como los de estigmatización de la pobreza o los de costes de información y gestión. Además, los límites presupuestarios y los problemas de gestión provocan que el periodo medio de concesión de la prestación se haya reducido en los últimos años, hasta los 5,64 meses en 2016.

\section{El nuevo programa de rentas mínimas de la CV: la Renta Valenciana de Inclusión (RVI)}

\subsection{Descripción de la renta valenciana de inclusión}

Constatada la insuficiencia del programa de renta mínima, la Generalitat Valenciana impulsó una reforma de la renta garantizada de la ciudadanía (RGC), a través de la nueva renta valenciana de inclusión (RVI), que se materializó con la aprobación por parte de la Generalitat Valenciana de la Ley 19/2017 y el Decreto 60/2018. ${ }^{5}$

La RVI es un instrumento más ambicioso que la RGC. De hecho, regula cuatro prestaciones distintas: la Renta de Garantía de Inclusión Social (RGIS), la Renta de Garantía de Ingresos Mínimos (RGIM), la Renta Complementaria de Ingresos del Trabajo (RCIT) y la Renta Complementaria de Ingresos por Prestaciones (RCIP). De todas ellas, solo la primera (RGIS) sería comparable con la RGC.

Una de las novedades más importantes es que la RGIS se introduce como un derecho subjetivo. Es decir, su concesión no dependerá de la previsión

4. En 2017 la cuantía anual máxima percibida por un hogar unipersonal era $385 € \times 6$ meses $=2.310 €$. Esta cantidad está muy por debajo del umbral de pobreza severa $(30 \%$ de la renta mediana) de la CV en 2017: 3.558,30 €. Al aumentar el tamaño del hogar, la diferencia es todavía mayor

5. Ley 19/2017, de 20 de diciembre, de la Generalitat, de Renta Valenciana de Inclusión (DOGV núm. 8196 de 22.12.2017) y Decreto 60/2018, de 11 de mayo, del Consell, por el que se desarrolla la Ley 19/2017, de 20 de diciembre, de la Generalitat, de Renta Valenciana de Inclusión (DOGV núm. 8310 de 05.06.2018). 
presupuestaria, como ocurre con la RGC, sino que debe concederse a todo hogar que cumpla los requisitos durante el periodo que sea necesario. Por otro lado, se suaviza uno de los requisitos generales ${ }^{6}$ de acceso a la prestación y se exige únicamente la residencia efectiva durante doce meses en lugar de la residencia legal.

Respecto a la cantidad teórica de la prestación, esta aumenta de manera sustancial para todos los casos. Mientras que la cuantía teórica de referencia mensual para una persona era del $62 \%$ del Indicador Público de Renta de Efectos Múltiples (IPREM) (385€) en la RGC, en la RGIS dicha cuantía es del $70 \%$ del SMI (515€). ${ }^{7}$ La RGIS también mejora la escala de equivalencia implícita utilizada para aumentar la cuantía teórica de la prestación conforme aumenta el tamaño del hogar (de un $3 \%$ adicional por persona de la RGC a un $8-12 \%$ de la RGIS). Sin embargo, la RGIS crece hasta un tamaño de hogar de seis miembros, mientras que la prestación de la RGC lo hacía hasta las trece personas. En cualquier caso, la cuantía teórica de la RGIS se mantiene siempre por encima de la RGC independientemente del tamaño del hogar. Conviene remarcar que esta cantidad teórica no tiene por qué coincidir con la cantidad efectivamente percibida en ambas prestaciones, ya que de este importe deben descontarse los ingresos percibidos previamente por el hogar.

En ambos casos, los titulares de la prestación deben firmar un acuerdo individual de inclusión. Este acuerdo incluye acciones específicas adaptadas a las necesidades particulares de cada individuo, que pueden ser de carácter personal, familiar, educativo, social y laboral, cuyo objetivo es prevenir o mejorar la situación o el riesgo de exclusión. Además, la nueva prestación prevé un incremento de hasta un $25 \%$ del importe reconocido para aquellos titulares que tengan que sufragar los gastos de alquiler o hipoteca de su vivienda habitual. En la siguiente tabla se resumen las principales características de ambas prestaciones.

Pero como hemos comentado, la nueva RVI es un instrumento más completo de garantía de ingresos e inclusión social. Como se observa en la tabla 4, incluye cuatro prestaciones, agrupadas en dos modalidades. Por un lado, las rentas de garantía, que incluyen la mencionada RGIS y además la Renta de Garantía de Ingresos Mínimos (RGIM). Estas dos rentas se pueden solicitar desde el 23 de abril de 2018. Por otro lado, la renta complementaria de ingresos, que comprende la Renta Complementaria de Ingresos del Trabajo (RCIT) y la Renta Complementaria de Ingresos por Prestaciones (RCIP). La aplicación de estas dos prestaciones se retrasará en el tiempo: un año para la RCIP (abril de 2019) y tres para la RCIT (abril de 2021). En cualquier caso, el objetivo último del sistema es que cualquier hogar pueda acceder a unos ingresos mínimos, no importa si recibe otras prestaciones o ingresos del trabajo.

6. Los requisitos generales de acceso a la RVI son los siguientes: los ingresos mensuales no pueden superar la cantidad teórica establecida en función del tamaño del hogar; estar empadronados o tener residencia efectiva durante doce meses en la Comunidad Valenciana; y tener cumplidos 25 años.

7. En todo el trabajo se presentan las referencias al SMI correspondientes al año 2018, cuando se comienza a implantar la nueva RVI. 
Tabla 3. Sistema valenciano de protección social: de la RGC a la RVI

\begin{tabular}{ll}
\hline $\begin{array}{l}\text { Renta Garantizada de la Ciudadanía (RGC) } \\
\text { 2008-2017 }\end{array}$ & $\begin{array}{l}\text { Renta de Garantía Inclusión Social (RGIS) } \\
\text { 2018- }\end{array}$ \\
\hline - Cuantía teórica mensual (1 persona): & - Cuantía teórica mensual (1 persona): \\
62\% IPREM = $385 €$ & - Escala de equivalencia: \\
- Escala de equivalencia: & $+8-12 \%$ por persona (hasta 6): $809 €$ \\
$\quad+3 \%$ por persona (hasta 13): $522 €$ & - Periodo máximo: indefinido \\
- Periodo máximo: seis meses & - Derecho subjetivo \\
- Prestación restringida presupuestariamente & - Firma de acuerdo de inclusión social \\
- Firma de acuerdo de inclusión social & - Incluye la residencia efectiva \\
- Residencia legal & $-+25 \%$ por alquiler o hipoteca \\
&
\end{tabular}

Fuente: elaboración propia.

Tabla 4. Tipo de prestaciones de la RVI, principales características

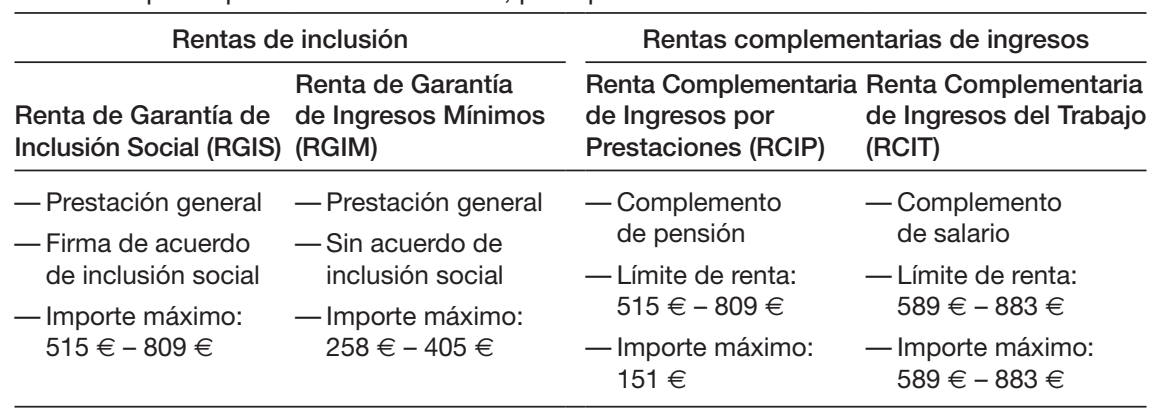

Fuente: elaboración propia.

La Renta de Garantía de Ingresos Mínimos (RGIM) es una nueva prestación. La principal diferencia con la RGIS es que esta prestación puede obtenerse cuando no se suscriba el acuerdo de inclusión social. Las cuantías de esta prestación oscilan entre $258 €$, para un hogar de una persona; y $405 €$, para hogares de seis o más personas. Es decir, el importe es la mitad del concedido a través de la RGIS. El objetivo de esta renta es hacer llegar una cuantía mínima de supervivencia a ciertos colectivos cuya inclusión social sea muy complicada.

La Renta Complementaria de Ingresos por Prestaciones (RCIP) se concederá si las prestaciones no contributivas recibidas por el hogar no alcanzan un determinado nivel, calculado con relación al SMI. Este nivel es exactamente el mismo que en la RGIS y varia entre $515 €$ para hogares unipersonales y $809 €$ para hogares de seis o más miembros. En este caso, la prestación suplirá la diferencia hasta alcanzar estos importes, con un tope máximo de $151 €$.

La Renta Complementaria de Ingresos del Trabajo (RCIT) se concederá si los ingresos del trabajo recibidos por el hogar no alcanzan un determinado nivel, calculado con relación al SMI. En hogares unipersonales el umbral es el 
$80 \%$ del SMI (589 €), pero puede alcanzar el $120 \%$ del SMI (883€) en hogares con seis o más miembros. La prestación suplirá la diferencia hasta alcanzar estos importes, aunque, de forma distinta a la RCIP, no está limitada por un tope máximo. La normativa no excluye expresamente a los trabajadores por cuenta propia, por lo que entendemos que los trabajadores autónomos podrán recibir en esta prestación cuando esté disponible.

\subsection{Proceso de cálculo para la estimación de la nueva RVI}

En el presente apartado se realiza una evaluación de la RVI que permita conocer a priori los efectos directos que puede tener esta medida. Para ello, estimamos el número de potenciales titulares y beneficiarios de la RVI, así como la cuantía que percibiría cada uno de ellos. Estos resultados permitirán comparar la RVI con la RGC y conocer sus efectos diferenciales en términos de pobreza y desigualdad.

En el proceso de cálculo, las cuatro modalidades de prestación que contempla la RVI son tratadas de manera separada. Para simplificar, agruparemos los cálculos en dos procesos: por una parte, las rentas de garantía (RGIS y RGIM); $y$, por otra, las rentas complementarias (RCIP y RCIT).

\subsubsection{Estimación de las rentas de garantía: RGIS y RGIM}

Para la estimación de las dos rentas de garantía, se parte de un primer supuesto (que luego se abandona): todos los perceptores suscriben el acuerdo de inclusión social y, por lo tanto, todos reciben la RGIS. En dicha estimación seguimos los siguientes pasos:

1. Estimación de la RGIS con datos (titulares, beneficiarios, nivel de ingresos) de la RGC de 2015, pero incorporando los nuevos parámetros establecidos. Para ello, recurrimos a los microdatos proporcionados por la Conselleria de Igualdad y Políticas Inclusivas. ${ }^{8}$ En este caso, el número de titulares se mantendría en 15.929 personas, pero la prestación mensual media se incrementaría de $393,50 €$ a 566,72 €.

2. Actualización de datos a 2017, concretamente el número de titulares. La Generalitat Valenciana proporciona el nuevo número de titulares correspondiente a este año, que aumenta hasta un total de 21.368 personas. Sin embargo, al no disponer de microdatos de todos los titulares de este año, se supone la misma prestación mensual media obtenida en 2015 (566,72 €).

3. Integrar a los empadronados sin residencia legal. A partir de los datos del INE, se calcula la diferencia entre el número de empadronados y el de residentes en la $\mathrm{CV}$ por nacionalidades y así se obtienen los nuevos titu-

8. Estos microdatos fueron elaborados y entregados por todas las comunidades autónomas al Ministerio de Sanidad, Servicios Sociales e Igualdad para la elaboración del informe «El sistema de garantía de ingresos en España: tendencias, resultados y necesidades de reforma", que fue coordinado por José Manuel Fresno (Ayala et al., 2016). 
lares potenciales. Suponemos que la tasa de cobertura de estas personas se mantiene en el mismo porcentaje que el resto de extranjeros en el año 2015 $(1,88 \%)$. Para mantener el mismo porcentaje de cobertura, hemos tenido en cuenta dos efectos contrapuestos. Si bien es cierto que la mayoría de residentes no legales tienen unos ingresos más bajos que el resto de residentes extranjeros, también deben ser más reacios a solicitar la prestación al estar en una situación irregular. Tras incluir a estas personas, la RGIS alcanzaría a un total de 22.341 titulares.

4. Introducir el cambio en la duración de la prestación. Partimos de suponer que cada titular recibirá la prestación durante los doce meses del año. Sin embargo, es necesario tener en cuenta que habrá titulares que dejarán de cobrar la prestación durante el año, debido a que se incorporarán al mercado laboral, y también habrá hogares que dejarán de cumplir los requisitos de la prestación por diversas circunstancias. Aunque es difícil estimar cuántas personas estarán en esta situación, hemos reducido el número de meses un $10 \%$, estimación que coincide con los datos de la RGC de 2015. Por lo tanto, en lugar de considerar un periodo de cobro anual de doce meses, tomaremos como referencia el $90 \%$, es decir, 10,8 meses.

5. Mayor alcance de la RGIS, debido a que el umbral de referencia es superior con la RGIS que con la anterior RGC. Al no tener información sobre los futuros nuevos perceptores de la prestación, simulamos su situación a partir de la Encuesta de Condiciones de Vida (ECV) ${ }^{9}$ de 2016. Para esta simulación, se parte de la renta disponible de cada hogar. Si esta renta declarada en la encuesta es inferior al umbral establecido, se ha considerado que ese hogar es un posible receptor de la RGIS y se ha calculado su importe como diferencia entre ambas cantidades. De estos posibles beneficiarios, se eliminan los hogares que tienen ingresos del trabajo, de prestaciones no contributivas y de desempleo, ya que la nueva ley establece la incompatibilidad con estas prestaciones. Con esta estimación, aparecerían 14.060 nuevos titulares de la RGIS, aunque su prestación media también se vería reducida, ya que su renta está mucho más próxima al umbral.

6. Tasa de cobertura. En el apartado anterior se ha supuesto una tasa de cobertura del $100 \%$ tras la introducción de la nueva RGIS. Sin embargo, en este tipo de prestaciones, un elevado número de potenciales beneficiarios finalmente no solicita la ayuda. Se trata del problema conocido como baja participación o, en terminología inglesa, non take-up. Los principales motivos que se aducen en la literatura para justificar esta situación son tres: el efecto estigmatizador asociado a la prestación, la dificultad para conseguir información sobre las ayudas para los colectivos más necesitados

9. Esta encuesta recoge datos relativos a los ingresos de los hogares y también a sus características socioeconómicas, y aporta información sobre educación, salud, situación laboral, vivienda y exclusión social. Elaborada por el Instituto Nacional de Estadística (INE). Incluye un total de 36.380 individuos para España, de los que 2.415 corresponden a la Comunidad Valenciana. 
y los costes de transacción que hay que asumir para conseguir la prestación (desplazamientos, documentación, etcétera). Para el cálculo de dicha tasa, nos basamos en los datos que conocemos de la RGC en 2017 (21.368 personas) y dividimos esta cantidad entre el número de potenciales titulares que se derivaría de la ECV (42.447 personas). Tras esta operación, obtenemos una tasa de cobertura del 50,34\%, que mantenemos constante para los nuevos titulares de la RGIS. Según nuestros cálculos, el número final de titulares aumentaría en 7.078 personas, hasta alcanzar una cifra final de 29.419 personas.

7. Incorporación del incremento de prestación debido a la existencia de gastos de alquiler o hipoteca de vivienda. A partir de la ECV de 2016, calculamos los perceptores de la RGIS que tienen que realizar pagos por alquiler o hipoteca, que alcanzan 12.836 hogares, lo que se refleja en un ligero incremento del presupuesto.

Una vez estimada la RGIS, debemos relajar el supuesto de que todos los solicitantes van a suscribir el acuerdo de inclusión social. Algunos de ellos preferirán recibir la mitad de dinero (RGIM) a cambio de no comprometerse con la Administración. Para conocer cuántos solicitantes firmarán el acuerdo, recurrimos a la Conselleria, que, por su experiencia en la gestión de la prestación, estima que el $90 \%$ de los solicitantes preferirá suscribir dicho acuerdo (Dirección General de Inclusión Social, 2016). Por lo que el número de titulares de cada prestación sería de 26.477 en la RGIS y de 2.942 en la RGIM.

\subsubsection{Estimación de las rentas complementarias de ingresos: $R C I P$ y $R C I T$}

A partir de abril de 2019, los perceptores de pensiones contributivas podrán complementar su importe con la RCIP, mientras que en abril de 2021 lo harán los perceptores de rentas del trabajo con la RCIT. El proceso de cálculo de estas dos prestaciones es el siguiente:

1. Estimación de los beneficiarios potenciales totales. Para este primer paso, se ha tenido que recurrir de nuevo a la información de la ECV, dado que no contamos con ficheros administrativos de estos tipos de hogares. Para el caso de la RCIP, se seleccionan los hogares que reciben prestaciones no contributivas por jubilación o invalidez que no superen los límites fijados por la normativa. Para el caso de la RCIT, se seleccionan aquellos hogares que perciben rendimientos netos del trabajo y de actividades económicas inferiores a los establecidos legalmente. Con esta simulación, se obtiene que para la RCIP habrá 19.335 titulares y para la RCIT 101.240.

2. Aplicación de la tasa de cobertura. Como ya se ha indicado anteriormente, debido al problema del non take-up, no todos los potenciales beneficiarios solicitan la prestación. En este caso, suponemos que se mantiene la tasa de cobertura del 50,34\%, con un ascenso del número de titulares de 9.733 personas en el caso de la RCIP y de 50.964 para la RCIT. 


\subsection{Principales resultados de la estimación de la renta valenciana de inclusión}

A continuación, se resumen los principales resultados de las estimaciones que hemos llevado a cabo sobre las cuatro modalidades de la renta valenciana de inclusión. Dado que resulta imposible conocer cómo va a variar la situación socioeconómica de los hogares durante los próximos años, hemos aplicado la normativa y la información sobre las cuatro prestaciones de la RVI a los microdatos de la ECV 2016. Es decir, no podemos calcular de forma precisa qué ocurrirá con la RVI en el futuro, pero sí podemos estimar qué hubiese ocurrido si la RVI se hubiese implantado completamente en 2016 teniendo en cuenta las características de la población valenciana de ese año.

Tabla 5. Principales resultados de la Renta Valenciana de Inclusión (50,34 \% de cobertura)

\begin{tabular}{lrrrrr}
\hline & RGIS & RGIM & RCIP & RCIT & TOTAL \\
\hline Titulares & 26.477 & 2.942 & 9.733 & 50.964 & 90.116 \\
Otros beneficiarios & 30.998 & 4.119 & 10.346 & 83.744 & 129.207 \\
Beneficiarios totales & 57.475 & 7.061 & 20.079 & 134.708 & 219.323 \\
Prestación media mensual & 498,33 & 279,93 & 109,88 & 186,05 & 272,64 \\
Prestación media anual & 5.382 & 3.023 & 1.187 & 2.009 & 2.944 \\
Inversión total & 142.497 .593 & 8.894 .082 & 11.549 .780 & 102.404 .925 & 265.346 .379 \\
\hline
\end{tabular}

RGIS: renta de inserción social; RGIM: renta de garantía de ingresos mínimos; RCIP: renta complementaria de ingresos por prestaciones; RCIT: renta complementaria de ingresos del trabajo.

Fuente: elaboración propia con los datos facilitados por la Conselleria de Igualdad y Políticas Inclusivas y la ECV 2016.

\section{Estimación de los efectos sobre la pobreza y la desigualdad}

En este epígrafe nos preguntamos por el impacto que tendrá la nueva RVI sobre los principales indicadores de pobreza y desigualdad.

\subsection{Pobreza monetaria}

El estudio de la pobreza se puede realizar desde diferentes perspectivas que incluyen múltiples indicadores (Ayala, 2004a). Sin embargo, en el presente trabajo se utiliza la perspectiva más habitual, que se refiere a la pobreza monetaria, pues los datos disponibles no permiten estimar otros indicadores de exclusión o de privación material, ni tampoco indicadores combinados como el AROPE.

Para el estudio de la pobreza monetaria, se utiliza la base de datos de la que se obtienen los indicadores oficiales utilizados en España y en el resto de la Unión Europea: la Encuesta de Condiciones de Vida de 2016. Como se ha simulado la RVI utilizando esta misma encuesta, se puede conocer la situación de pobreza monetaria que había en 2016, cuando se percibía la RGC, y también la nueva tasa de pobreza que existiría ese mismo año si la renta de los hogares se modificara únicamente en el importe de la RVI. De esta forma, 
podemos aislar el efecto que tendrá la RVI sobre la pobreza manteniendo constantes el resto de características sociales y económicas de la población. También conviene aclarar que el efecto sobre la pobreza será el derivado directamente de las prestaciones de la RVI, sin considerar los efectos indirectos que la nueva prestación puede tener sobre otras variables económicas.

Para representar la pobreza monetaria en países desarrollados, se suelen utilizar indicadores relativos, que comparan la renta de las personas más vulnerables con un umbral de pobreza que suele establecerse en función de la media o la mediana de la renta de la población. En la Unión Europea, el umbral de pobreza más habitual es el $60 \%$ de la mediana de cada país, que es el que calcula el Instituto Nacional de Estadística mediante los datos de la ECV. Este umbral estaría reflejando la situación de la población en riesgo de pobreza, pero no de pobreza extrema o severa.

La RVI, al igual que otras rentas mínimas de inserción, no favorece a toda la población en riesgo de pobreza, sino solamente a los hogares más necesitados que no han podido obtener otras prestaciones públicas y están en situación de pobreza severa. Por este motivo, en este análisis se utiliza el umbral de pobreza severa para analizar si la RVI permite a sus beneficiarios salir de esta grave situación. Aunque existen diferentes umbrales de este tipo, se va a emplear el más extendido, que corresponde al $30 \%$ de la renta mediana de la población.

En este punto también se deben tomar dos decisiones metodológicas. La primera se refiere a la escala de equivalencia que se va a utilizar. Sobre esta materia no parece haber discusión, ya que todos los resultados sobre pobreza oficiales de los países europeos emplean la escala de equivalencia de la OCDE modificada, que da un valor 1 al primer adulto del hogar, 0,5 al resto de adultos y 0,3 a los menores de 16 años. En este caso, seguiremos esta escala de equivalencia para presentar los resultados sobre pobreza severa. La segunda decisión se refiere al umbral empleado, ya que se puede utilizar el umbral obtenido para toda España (4.104,25 € anuales en 2016) o bien el umbral de los residentes en la Comunidad Valenciana (3.558,30 € anuales en 2016). Las dos alternativas serían válidas, pero al ser una política llevada a cabo desde el Gobierno autonómico, cobra más sentido comparar la renta de los beneficiarios con el resto de los habitantes valencianos, por lo que nos decantamos por el umbral autonómico (Zugasti y Laparra, 2017). Este umbral se incrementará en función de las personas que convivan en el hogar, según la escala de equivalencia de la OCDE. En la tabla 6 se presentan los umbrales para los tipos de hogar más frecuentes.

Una vez definidos los umbrales a utilizar, se calcula un indicador de incidencia de la pobreza. El más habitual es la ratio de pobreza, que consiste en dividir el número de personas situadas por debajo del umbral entre el total de la población. En este caso, nos tendríamos que preguntar cuántas personas se situaban por debajo de este umbral antes de percibir la RVI (renta inicial) y cuántas se situarían tras recibir la prestación (renta final). En la tabla 7 se muestran los efectos estimados sobre la pobreza severa de la renta valenciana. 
Tabla 6. Umbrales de pobreza severa para diferentes tipos de hogar

\begin{tabular}{lc}
\hline Tipo de hogar & Umbral de pobreza severa \\
\hline 1 adulto & $3.558,30$ \\
1 adulto +1 niño & $4.625,79$ \\
1 adulto +2 niños & $5.693,28$ \\
1 adulto +3 niños & $6.760,77$ \\
2 adultos & $5.337,45$ \\
2 adultos + 1 niño & $6.404,94$ \\
2 adultos + 2 niños & $7.472,43$ \\
2 adultos +3 niños & $8.539,92$ \\
3 adultos & $7.116,60$ \\
3 adultos + 1 niño & $8.184,09$ \\
3 adultos + 2 niños & $9.251,58$ \\
3 adultos + 3 niños & $10.319,07$ \\
\hline
\end{tabular}

Fuente: elaboración propia a partir de la ECV 2016.

Tabla 7. Efecto sobre la pobreza severa de la RVI

\begin{tabular}{lcc}
\hline & Renta inicial & Renta final \\
\hline Ratio de pobreza & $5,14 \%$ & $2,98 \%$ \\
Número de pobres & 251.702 & 145.817 \\
Reducción de pobres & & 105.886 \\
$\%$ de reducción & & $42,1 \%$ \\
\hline
\end{tabular}

Fuente: elaboración propia a partir de la ECV 2016.

Antes de comenzar a analizar los resultados de la RVI, conviene aclarar qué significado tiene la renta inicial que se emplea como punto de partida. Según los microdatos que figuran en la ECV 2016, el 5,14\% de toda la población residente en la Comunidad Valenciana está en una situación de pobreza severa, lo que afecta a 251.702 personas. Estas personas ya habrán recibido todas las ayudas públicas existentes, incluida la propia RGC, pues los resultados se refieren a la renta disponible del hogar después de prestaciones.

En este punto es importante preguntarse si la anterior RGC ha permitido que alguna persona haya podido salir de la pobreza extrema durante 2016. La respuesta es negativa. La RGC tiene una cuantía máxima mensual de $385 €$ en hogares de una persona, que, multiplicada por las seis mensualidades máximas, lleva a un importe anual máximo de $2.311 €$ por titular. Esta cuantía se sitúa muy por debajo del umbral de pobreza severa para un hogar de una persona (3.530,70€). Si bien es cierto que en hogares más amplios la RGC es más generosa, tampoco se va a superar nunca el umbral, ya que este aumenta más rápidamente al tener en cuenta la escala de equivalencia. Con este comentario no se quiere restar importancia a la RGC. Aunque su efecto sobre la incidencia de la pobreza severa ha sido nulo, no hay que desdeñar su relevancia sobre la intensidad de la pobreza. Es cierto que habrá el mismo número de personas pobres, pero ahora serán menos pobres, ya que su renta estará más cercana al umbral. 
En cuanto al efecto de la RVI sobre la reducción de la pobreza, puede comprobarse que es mayor al aumentar la tasa de cobertura, con una disminución de la tasa de pobreza severa del 5,14 \% al 2,98 \%, que supondría que 105.886 personas dejarían de ser consideradas pobres, lo que implicaría una reducción de la pobreza severa de un $42,1 \%$.

Aunque una reducción de la pobreza de 105.886 personas puede considerarse como un gran logro de la RVI, también conviene subrayar que la pobreza severa estaría todavía muy lejos de desaparecer, ya que quedarían todavía 145.817 personas en esta situación. Tres son los motivos por los que continuaría habiendo personas en riesgo de pobreza severa tras recibir la RVI.

a) La RVI no llega a todas las personas en situación de pobreza severa, sino solo a una parte de ellas, debido al problema del non take-up, que presume una tasa de cobertura lejana al $100 \%$. Si la tasa de cobertura se mantiene en el 50,34\%, únicamente ese porcentaje de las personas pobres recibiría la prestación. También es cierto que, si el Gobierno valenciano consigue alcanzar una tasa de cobertura superior, los niveles de pobreza se reducirán sensiblemente. Para intentar minimizar este problema, la labor de los servicios sociales municipales puede ser fundamental en el futuro, si tratan de contactar con las personas en situación de pobreza severa y desempeñan una labor de asesoramiento y de reducción de los trámites burocráticos.

b) Existen hogares que están por debajo del umbral de pobreza severa en términos de renta, pero que no van a ser receptores de RVI por diversos motivos administrativos. Entre estos motivos, el que consideramos más importante es la percepción de prestaciones por desempleo. Al tratarse de prestaciones incompatibles con la RVI, muchos desempleados cobrarán una cantidad inferior por desempleo que la que les correspondería de renta de inclusión, lo que les impide salir de la situación de pobreza. En nuestra opinión, esta incompatibilidad debería suprimirse para no penalizar a los desempleados con derecho a prestación.

c) El tercer motivo es técnico y tiene que ver con las escalas de equivalencia implícitas en la RVI, que suponen un incremento entre el $8 \%$ y el $15 \%$ por cada miembro adicional del hogar. Sin embargo, la escala de equivalencia que se utiliza en las estadísticas sobre pobreza es la de la OCDE modificada, que supone un incremento del $50 \%$ para los adultos adicionales y del $30 \%$ para los niños. En otras palabras, los hogares unipersonales siempre saldrán de la situación de pobreza gracias a la RVI, pero no ocurrirá siempre lo mismo con los hogares más numerosos, especialmente en aquellos donde convivan tres o más personas adultas.

\subsection{Desigualdad}

Los análisis de desigualdad son importantes en la evaluación de políticas públicas porque permiten saber si la política analizada ha llevado a una distribución de la renta más o menos equitativa que la que existía en el punto de partida. Si 
Tabla 8. Efecto sobre la desigualdad de la RVI

\begin{tabular}{lcc}
\hline & Renta inicial & Renta final \\
\hline Índice de Gini & $32,43 \%$ & $31,78 \%$ \\
$\%$ de reducción & & $2,0 \%$ \\
\hline
\end{tabular}

Fuente: elaboración propia a partir de la ECV 2016.

bien es cierto que la RVI va a tener cierto efecto sobre la desigualdad, ya que los más necesitados van a recibir una prestación, este efecto no cabe esperar que sea muy relevante, pues la mayoría de la población quedará exactamente en la misma situación en la que estaba antes de existir la prestación. Si se quiere conseguir una reducción significativa de la desigualdad, es más adecuado utilizar otro tipo de políticas públicas, tanto de ingresos como de gastos, que tengan en cuenta a la mayor parte de la población.

Para evaluar la desigualdad, se pueden utilizar distintos índices, como Atkinson o Theil, aunque el más empleado sigue siendo el índice de Gini, que se basa en la curva de Lorenz. Este índice toma valores entre 0 y 1 . El valor 0 representaría la igualdad perfecta, en la que todos los hogares tendrían la misma renta equivalente, mientras que el valor 1 equivaldría a la desigualdad completa, en la que un solo hogar tendría toda la renta de la sociedad.

En la tabla 8 se muestran los resultados que se han obtenido utilizando la ECV 2016. Al igual que se ha hecho en el análisis de la pobreza, se ha representado la desigualdad en la renta inicial y en la final teniendo en cuenta las cuatro prestaciones de la RVI. Los resultados, aunque de escasa cuantía, son claros y muestran una pequeña disminución de la desigualdad derivada de la RVI. El índice de Gini apenas mejora un $2 \%$.

\section{Conclusiones}

A lo largo de este trabajo hemos analizado las previsibles consecuencias derivadas de la introducción de la renta valenciana de inclusión. Se parte de un diagnóstico que incluye dos elementos. El primero alude al contexto socioeconómico de los últimos años. La principal conclusión es que la crisis ha golpeado muy duramente a las capas más empobrecidas de la ciudadanía. Además, la pobreza se ha extendido, al incorporar a colectivos de trabajadores y pensionistas. Los daños han sido en general mayores en la Comunitat Valenciana que en el resto de España. El segundo elemento hace referencia a la evolución de la entonces vigente Renta Garantizada de la Ciudadanía. Ante la crisis económica, la prestación ha respondido aumentando el gasto y el número de beneficiarios. Sin embargo, esta prestación muestra ciertos síntomas de agotamiento: sus niveles son insuficientes y se mantienen muy alejados de la media española, tanto en gasto anual como en tasa de cobertura. También muestra la desatención a colectivos que tradicionalmente no habían experimentado riesgo de pobreza y exclusión (trabajadores pobres y pensionistas). Estos dos elementos aconsejan un replanteamiento del diseño de la renta mínima valenciana. 
Nuestro objetivo ha sido analizar si la nueva renta valenciana de inclusión puede mejorar esta situación. Después de la descripción de sus principales características y componentes, hemos realizado una estimación detallada de su impacto utilizando técnicas de microsimulación. El principal resultado de nuestras estimaciones es que la nueva prestación supondrá un coste mucho mayor para el Gobierno valenciano, pero también alcanzará a muchas más personas y se situará como una de las rentas de inserción más generosas de nuestro país.

En cualquier caso, el resultado que más interesa destacar es el fuerte efecto que tendrá la introducción de la renta valenciana de inclusión sobre la pobreza y la desigualdad. Según los supuestos considerados, la prestación supondría una importante reducción de la pobreza severa. La nueva renta reduciría la tasa de pobreza desde un 5,14\% actual hasta un 2,98\%. Es decir, haría disminuir la pobreza en un $42,1 \%$, o sea que más de 105.000 personas saldrían de la situación de pobreza severa. La desigualdad también se verá reducida gracias a la nueva renta, aunque su efecto no es tan llamativo como en el caso de la pobreza.

Estos efectos positivos sobre la pobreza y la desigualdad podrían mejorarse si la RVI consiguiera eliminar, o al menos reducir, el problema del non take-up. La Administración valenciana y los servicios sociales municipales deberían realizar un esfuerzo para que esta prestación llegara a todas las personas que cumplen los requisitos. Además, convendría extender la prestación a ciertos colectivos vulnerables, como los trabajadores desempleados, que no tienen derecho a percibirla cuando están cobrando una prestación por desempleo. También sería deseable un tratamiento más generoso con las familias. El aumento de la prestación en los hogares con más de un miembro es insuficiente, según las escalas de equivalencia estándar.

Hay que añadir que todo el análisis está basado en datos del pasado y recordar que las rentas mínimas son extraordinariamente sensibles al ciclo económico. En el pasado, el incremento del gasto ha estado íntimamente relacionado con los peores años de la última crisis. Ante la incertidumbre actual sobre los efectos que la pandemia COVID-19 tendrá sobre la economía, tanto en su profundidad como en su duración, debemos tomar los resultados presentados en este trabajo como meramente orientativos. Sin embargo, estas prestaciones son especialmente decisivas en este tipo de situaciones. Al tratarse de la última «red de seguridad económica», son prestaciones muy útiles ante coyunturas como la actual, ya que responden con mucha agilidad.

\section{Financiación}

El presente trabajo ha recibido el apoyo institucional del Proyecto HIECPU/2019/2 de la Conselleria de Hacienda y Modelo Económico de la Generalitat Valenciana. 


\section{Referencias bibliográficas}

Arriba, Ana (1999). "Procesos de implantación de políticas de rentas mínimas de inserción en España». Papeles de Economía Española, 81, 219-241.

- (2014). «El papel de la garantía de mínimos frente a la crisis. VII Informe sobre exclusión y desarrollo social en España 2014». Madrid: Fundación Foessa. Documento de trabajo 5.7. Disponible en: <http://www.foessa2014.es/informe/uploaded/documentos_trabajo/04112014003757_2107.pdf>.

Ayala, Luis (2004a). «Las políticas contra la pobreza (I): Delimitación». En: Gimeno Ullastres. J. A. (ed.). Exclusión social y estado de bienestar. Madrid: Centro de Estudios Ramón Areces.

- (2004b). «Las políticas contra la pobreza (II): Experiencias europeas y realidad española». En: Gimeno Ullastres, J. A. (ed.). Exclusión social y estado de bienestar. Madrid: Centro de Estudios Ramón Areces.

Ayala, Luis; Arranz, José María; García, Carlos; Martínez, Lucía (2016). El sistema de garantía de ingresos en España: tendencias, resultados y necesidades de reforma. Madrid: informe programa PROGRESS. Disponible en: <http://www.carm.es/ ctra/cendoc/haddock/17014.pdf>.

Bergantiños, Noemí; Font, Raquel; Bacigalupe, Amaia (2017). «Las rentas mínimas de inserción en época de crisis. ¿Existen diferencias en la respuesta de las comunidades autónomas?». Papers. Revista de Sociologia, 102 (3), 399-420. $<$ https://doi.org/10.5565/rev/papers.2315>.

Consejo Económico y Social (2017). Políticas públicas para combatir la pobreza en España (primera edición ed.). Consejo Económico y Social. Disponible en: <http:// www.econis.eu/PPNSET?PPN=893745898>.

Dirección General de Inclusión Social (2016). Informe económico de la ley de renta valenciana de inclusión. Valencia: Generalitat Valenciana.

Dirección General de Política Social (2012). Informe Rentas Minimas de Inserción. Año 2011. Madrid: Ministerio de Sanidad y Política Social.

- (2013). Informe Rentas Minimas de Inserción. Año 2012. Madrid: Ministerio de Sanidad y Política Social.

- (2014). Informe Rentas Minimas de Inserción. Año 2013. Madrid: Ministerio de Sanidad y Política Social.

- (2015). Informe Rentas Minimas de Inserción. Año 2014. Madrid: Ministerio de Sanidad y Política Social.

- (2016). Informe Rentas Minimas de Inserción. Año 2015. Madrid: Ministerio de Sanidad y Política Social.

Fuenmayor, Amadeo; Granell, Rafael (2011). La política de lucha contra la pobreza y la exclusión social. Simulación y evaluación de las politicas estatales y autonómicas. Premio FIPROS. Ministerio de Empleo y Seguridad Social. Disponible en: <http:// www.seg-social.es/descarga/174220>.

Laparra, Miguel (2004). "La travesía del desierto de las rentas mínimas en España». Documentación Social, 135, 57-76.

Laparra, Miguel; Ayala, Luis (2009). El sistema de garantía de ingresos mínimos en España y la respuesta urgente que requiere la crisis social. Madrid: Fundación Foessa.

Lavia, Cristina; Moreno, Gorka; Aierdi, Xabier (2014). «Diferencias en el uso de los programas de rentas mínimas según nacionalidad. La Renta de Garantía de Ingresos en la Comunidad Autónoma vasca». Revista Internacional de Sociología, 72 (3), 633-660. <https://doi.org/10.3989/ris.2013.01.23>. 
Moreno, Luis; Matsaganis, Manos; Ferreira, Maurizio; Capucha, Luis (2003). «¿Existe una malla de seguridad en la Europa del sur? La lucha contra la pobreza y la exclusión en España, Grecia, Italia y Portugal». Revista Internacional de Sociologia, 61 (36), 7-31. <https://doi.org/10.3989/ris.2003.i36.312>.

Riba, Clara; Ballart, Xavier; Blasco, Jaume (2011). «Minimum Income and Labour Market Integration Processes: Individual and Institutional Determinants». Revista Española de Investigaciones Sociológicas, 133, 43-58. <https://doi.org/10.5477/cis/reis.133.43>.

Rodríguez-Cabrero, Gregorio (2009). Valoración de los programas de rentas mínimas en España. Bruselas: European Commission - DG Employment, Social Affairs \& Inclusion. Disponible en: <http://ec.europa.eu/social/BlobServlet?docId=9043\&langId=es $>$. (Consultado el 05/03/18).

Urteaga, Eguzki (2011). «Las rentas mínimas en Francia». Papers. Revista de Sociologia, 96 (3), 959-983. $<$ https://doi.org/10.5565/rev/papers/v96n3.209>.

Zugasti, Nerea; Laparra, Miguel (2017). «Midiendo la pobreza a nivel autonómico en España. Una propuesta reflexiva». Revista Española de Investigaciones Sociológicas, 158, 117-136.

<https://doi.org/10.5477/cis/reis.158.117>. 